\title{
Mesoscopic solvent simulations: Multiparticle-collision dynamics of three-dimensional flows
}

\author{
E. Allahyarov and G. Gompper \\ Institut für Festkörperforschung, Forschungszentrum Jülich, D-52425 Jülich, Germany
}

(Received 14 February 2002; published 11 September 2002)

\begin{abstract}
A recently developed mesoscopic solvent model with multiparticle-collision dynamics is applied to threedimensional solvent flows in a channel with and without a spherical obstacle. The advantage of a gravitationally driven flow of the solvent over the flow induced by a pressure gradient in the calculation of the solvent viscosity is demonstrated. Three different algorithms for stochastic collision steps are investigated and compared. In particular, we have examined an alternative algorithm with relative velocities drawn from a MaxwellBoltzmann distribution at each collision step. This algorithm increases the numerical efficiency of the mesoscopic model for solvent flows with low and intermediate Reynolds numbers. Our simulation results for the recirculation length of stationary vortices behind a spherical obstacle are in good agreement with the previous experimental measurements.
\end{abstract}

DOI: 10.1103/PhysRevE.66.036702

PACS number(s): $02.70 . \mathrm{Ns}, 47.11 .+\mathrm{j}, 82.20 . \mathrm{Wt}$

\section{INTRODUCTION}

Rheology, phase behavior, stabilization, and other physicochemical properties of supramolecular solutions are mainly governed by the effective interactions between solute particles. Many soft matter systems, such as colloids, polymers, or biological macromolecules are dispersed in a molecular solvent [1]. These systems typically consist of three components, which are the solute (macroion) particles, the solvent molecules, and small salt ions. When such a soft matter system is subjected to an external flow field, the effective solute-solute interactions are modified, and consequently the nature of the medium is changed. Since the dynamics of interest in such systems usually occurs on long time scales and over large distances, a direct and full simulation of such a complex fluid is a challenging task. Therefore, new mesoscale simulation techniques are necessary to study the dynamical behavior of these systems.

In contemporary theoretical models (integral equations theories, density functional theory, Poisson-Boltzmann-like theories) and numerical simulations the system is usually coarse grained into a two-component system consisting of large colloidal particles and small salt ions. The granular nature of the solvent is dropped whereas its electrostatic screening feature is taken into account through embedding the whole system into a dielectric bath continuum. Such coarse-grained models work very well for the static behavior of micrometer-sized colloids. For smaller particles, with diameters in the range of 1-10 nanometers, the discreteness of the electric charges and the granular nature of the solvent becomes important. The implementation of restricted models for the solvent (hard sphere fluid, dipolar fluid) in some applications to small colloidal systems [2-7] gives rise to pronounced changes in the system characteristics compared to those obtained previously within coarse-grained models.

The modeling becomes more complicated when the dynamical behavior is studied. For not too small colloidal particles, as an alternative to analytical approaches based on the Stokes approximation, one is led to consider mesoscopic models for the solvent dynamics which incorporate the essential dynamical properties, but are simple enough to be simulated for long times and on long distance scales, such as the lattice-Boltzmann method [8] or dissipative-particle dynamics [9]. A recently developed mesoscopic model, multiparticle-collision dynamics [10,11], was successfully applied in Ref. [12] to the two-dimensional (2D) flow around obstacles. Within this mesoscopic solvent method, which was further improved in Refs. [12,13], the fluid is modeled by point particles with velocities and positions as continuous variables. The dynamics is carried out synchronously via simultaneous rotation of relative velocities of all particles in a collision volume. It was shown that the dynamics satisfies the mass, momentum, and energy conservation laws and yields the correct hydrodynamic behavior [10]. Multiparticle-collision dynamics provides a very promising approach for studying the dynamical behavior of colloidal suspensions [11].

In this paper, the mesoscopic solvent model is applied to the three-dimensional solvent flow between planar walls and around a spherical obstacle. An advantage of a gravitationally driven flow over the flow forced by a pressure gradient, which has been used in the previous applications of multiparticle-collision dynamics, is addressed. We present a detailed investigation of the dependence of the solvent viscosity on the mesoscopic system parameters. The rest of the paper is organized as follows. In Sec. II we shortly describe the multiparticle-collision dynamics model. Section III is devoted to simulation details. Simulation results are presented and discussed in Sec. IV. Finally we conclude in Sec. V.

\section{THE MODEL}

$N$ point particles are distributed randomly inside a 3D simulation channel of volume $L_{x} \times L_{y} \times L_{z}$. The positions $\vec{r}_{i}$ and velocities $\vec{v}_{i}, i=1,2, \ldots, N$ of the particles are considered as continuous variables. During the time intervals $h(j$ $-1)<t<h j$, where $j$ are positive integer numbers, the system evolves via standard streaming, hence the new particle coordinates are

$$
\vec{r}_{i}(t)=\vec{r}_{i}(t-h)+h \vec{v}_{i}(t-h)
$$


At discrete times $t=h j$, stochastic collisions between solvent particles occur in the following way. First, the system is coarse grained into $n_{s}=x_{s} \times y_{s} \times z_{s}$ collisional cells of volume $L_{x} / x_{s} \times L_{y} / y_{s} \times L_{z} / z_{s}$. For simplicity we introduce a set of integer numbers $(a, b, c)$ such that $0 \leqslant a<x_{s}, 0 \leqslant b$ $<y_{s}, 0 \leqslant c<z_{s}$, which completely define the cell coordinates. For example, a cell with coordinates $(0,0,0)$ is in the bottom left corner of the simulation box, whereas a cell with coordinates $\left(x_{s}-1, y_{s}-1, z_{s}-1\right)$ is in the top right corner of the simulation box. Further, let the integer variable $p$ denotes the cell number, $p=(a, b, c)$. Clearly, the variable $p$ does not exceed the total number of collision cells $n_{s}$ in the simulation box. Suppose that, there are $n_{p}$ point particles in each of the collisional cells $p$. The average number (averaged over all collisional cells) of particles per cell is $<n_{p}>=N / n_{s}=M$. Second, we define the macroscopic velocity $\vec{u}_{p}$ in cell $p$ as

$$
\vec{u}_{p}=\sum_{i=1}^{n_{p}} \vec{v}_{i} / n_{p}
$$

Here $i$ runs over all the particles belonging to considered cell $p=(a, b, c)$. Third, the relative velocities of particles in each of the collision cells $p=1,2, \ldots, n_{s}$, defined as $\vec{v}_{i}-\vec{u}_{p}, i$ $=1, \ldots, n_{p}$, is stochastically rotated by an angle $\pm \alpha$ around a randomly directed three-dimensional vector $\vec{R}_{p}$. The procedure of stochastic rotations is described in details in the Appendix.

Finally, the new velocities of particles in cell $p$ are calculated as

$$
\vec{v}_{i}(t+h)=\vec{u}_{p}+\vec{\xi}_{i}(t)
$$

Here $\vec{\xi}_{i}$ is the relative velocity of particle $i$ after the rotation, see the Appendix.

In addition, we have examined two alternative algorithms for changing the particle velocities in the collision step. These algorithms, which we call "random angle method" and "random velocity method," were applied to solvent flow between planar walls and compared to the results of conventional stochastic rotational method. Within the "random angle method," a random set of rotational angles, $\alpha_{p}, p$ $=1, \ldots, n_{s}$, is generated prior to every collision step. Then, the angle $\alpha_{p}$ is used as a rotational angle in the stochastic rotation step for all particles pertaining to cell $p$. The "random velocity method" is assumed to be a fully stochastic procedure, with no explicit rotation of velocities. Instead, at each collisional step, we update the relative velocities $\vec{v}_{i}$ $-\vec{u}_{p}$ of particles in collisional cell $p$ by Maxwell-Boltzmann distributed velocities $\vec{V}_{i}$ with zero average, $\sum_{i=1}^{p} \vec{V}_{i} / n_{p}=0$. Hence the new velocities of particles in a cell $p$ after a collision time $t$ read

$$
\vec{v}_{i}(t+h)=\vec{u}_{p}+\vec{V}_{i}(t)
$$

During the collisional step, we adopted a random shifting procedure $[12,13]$ to ensure the Galilean invariance.

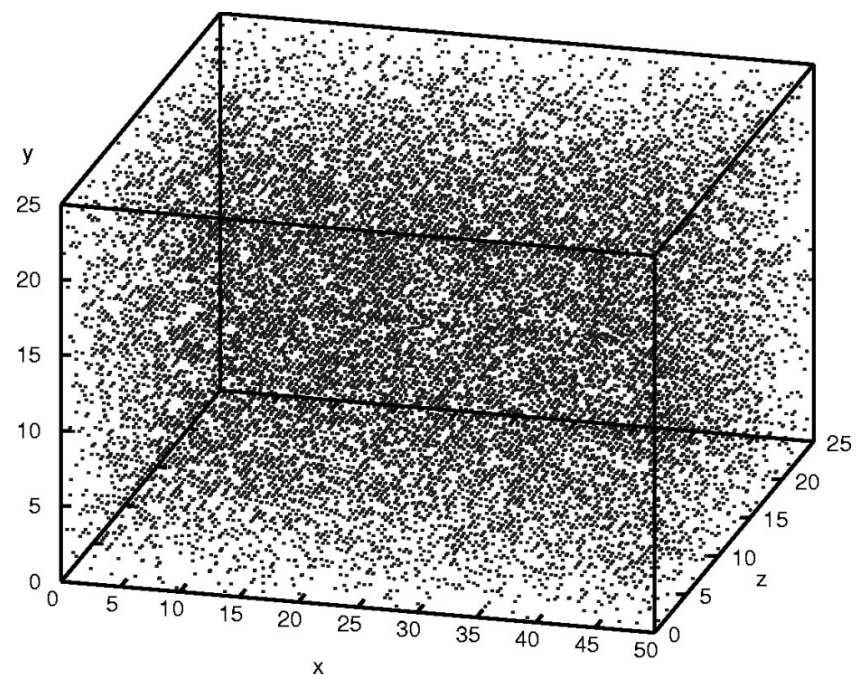

FIG. 1. Snapshot of the simulation box filled with point (solvent) particles under shear flow.

\section{SIMULATION DETAILS}

We consider a system consisting of $N=10^{6}$ point particles in a rectangular channel of volume $50 \times 25 \times 25$, see Fig. 1 . The longest side of the simulation box (channel) is taken to coincide with the $x$ direction of the system, and the mass of solvent particles is set to $m=1$. The boundary planes at the top, $y=0$, and bottom, $y=L_{y}=25$, of the simulation box are rigid walls with bounce-back [14] boundary conditions on their surfaces. In the $x$ and $z$ directions we apply periodic boundary conditions. At each collision step, the simulation box is divided into $50 \times 25 \times 25$ cubic cells, thus $x_{s}=50, y_{s}$ $=25, z_{s}=25, n_{s}=31250$ and collisional cell has a volume $1 \times 1 \times 1$. For partially filled cells, i.e., cells which cut the system walls, we employ the generalized bounce-back rule, developed in Ref. [12]. The system temperature $T$ and simulation time step $h$ define the dimensionless parameter $\Lambda$ $=l / l_{c}$, where $l=h \sqrt{k_{B} T}$ is the mean free path of solvent particles, $k_{B}$ is the Boltzmann constant, and $l_{c}=1$ is the linear cell size. The other system parameters are the average particle number per cell $M$ and the stochastic rotational angle $\alpha$. Accordingly, our goal is to investigate the influence of these parameters onto the macroscopically measurable control parameter of fluid - the viscosity $\eta$ of the mesoscopic solvent. In Ref. [13] some of these dependences were determined for a $2 \mathrm{D}$ system.

It is well established that the solvent viscosity can be numerically determined in the two different ways. The first way, related to the Green-Kubo expression [15], applies to systems in thermal equilibrium, but unfortunately suffers from large signal-to-noise ratio for the viscosity. The second way, which we follow in this paper, relates to systems in nonequilibrium conditions (systems with an induced flow) where the viscosity can be calculated with high precision. In this case, $\eta$ is derived from the stationary one-dimensional velocity field profile of forced flow, which gives

$$
\eta=-\frac{A L_{y}^{2}}{8 v_{\max }}
$$




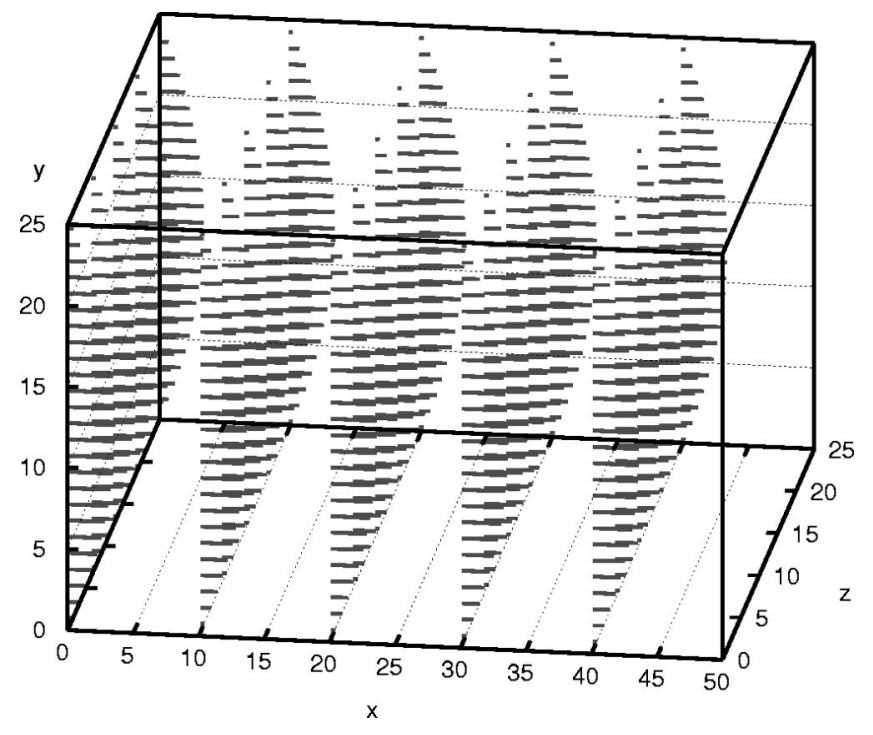

FIG. 2. A typical three-dimensional velocity profile for solvent Poiseuille flow between two parallel walls at $y=0$ and $y=25$.

Here $A=\left|\vec{\nabla}_{x} p\right|$ is an external field that produces the flow, $p$ is the thermodynamic pressure in the system, and $v_{\max }$ is the maximal flow velocity in the midplane of the channel. Equation (5) is a solution of the Navier-Stokes equation for Newtonian flow of an incompressible fluid with boundary conditions $v_{x}(y=0)=v_{x}\left(y=L_{y}\right)=0$. It is assumed that the external field $A$ is directed along the channel length ( $x$ direction in Fig. 1) and induces a Poiseuille flow.

There are several types of flow between parallel walls: forced, gravitational, and surface induced. The forced flow has been considered in Refs. [10,12,13], where the pressure gradient is mimicked by hanging a virtual pump to the inlet of the channel. Our test simulations, not shown here, reveal this method to have drawbacks - a deformation of the velocity-field and density profiles of the particles at the inlet and outlet of the channel, and a gradual density drop of particles along channel length. That is why any simulation within this forced method should be carried out for long channels and numerical measurements have to be done in the center of the channel, where system parameters are least disturbed.

The use of gravitationally driven flow, which is adopted in this paper, does not perturb the velocity-field profile of the particles and their density profile along channel length, but slightly disturbs the solvent density across the cross section of the channel. The main advantage of this flow is its simplicity and the possibility of direct molecular dynamic simulations without artificial tricks to suppress the inhomogeneities in velocity and density profiles. In this case, $A=\rho g$ in Eq. (5), where $\rho=M / l_{c}^{3}$ is the solvent density. The strength of the gravitational field can be varied by tuning the acceleration constant $g$. For fixed $g$, a steady Poiseuille flow quickly and self-consistently develops in the system, which is coupled to a thermostat [16] to keep the temperature $T$ constant during the simulation run, see Fig. 2.

\section{SIMULATION RESULTS AND DISCUSSION}

\section{A. Poiseuille flow and solvent viscosity}

We start with the discussion of the results with the influence of the stochastic rotational angle $\alpha$ on the particle density across the channel width. Hence we fix the strength of the external field at $g^{*}=g h / \sqrt{k_{B} T}=0.005$ and the average particle number per collisional cell to $M=32$. Simulation results for the solvent density across the channel width are plotted in Fig. 3 for three different values of $\Lambda=l / l_{c}$. There is a pronounced effect of $\alpha$ on the solvent density profiles $\rho(y)$. For small values of $\Lambda$, the density profiles $\rho(y)$ exhibit a common trend: with increasing $\alpha$, the solvent density at midplane $\rho\left(L_{y} / 2\right)$ decreases, whereas the density at the boundary walls, $\rho(0)$ and $\rho\left(L_{y}\right)$, increases. Less perturbed density profiles, estimated as the difference between its maximal and minimal values, correspond to angles greater than $90^{\circ}$ (strong collisions). For intermediate and large values of $\Lambda$, this trend becomes opposite. Now less perturbed profiles are seen for small values of $\alpha$. Such a behavior can be understood in the following manner. The parameters $\alpha$ and $\Lambda$ affect the solvent particle dynamics in different ways. The angle $\alpha$ is the characteristic parameter for interaction of particles within a cell. Note that the lower bound $\alpha=0$ corresponds to the case of an ideal solvent gas, i.e., the case of freely streaming particles. The upper bound $\alpha=180^{\circ}$, in opposite, implies very strong interaction between particles in a collision cell. The second parameter $\Lambda$ affects the correlations between particles or, in other words, is a measure of the strength of induced "molecular chaos" in the system. The larger $\Lambda$, the smaller the correlations between cell particles, since the collisional environment of each particle no longer depends on the instantaneous average velocity in a cell. These two parameters can be expected intuitively to be correlated. This is obvious from the curves for $\alpha=30^{\circ}$ in the upper plot and for $\alpha=150^{\circ}$ in the lower plot of Fig. 3. Both curves have almost identical shapes; whereas the first corresponds to weak "interaction" and strong "correlation," the second corresponds to strong "interaction" and weak "correlation." Note that the inhomogeneity for all curves in Fig. 3 is less than $10 \%$.

Surprisingly, both alternative collision algorithms discussed in Sec. II yield almost identical density profiles for the solvent particles. These profiles, displayed as dashed lines in Fig. 3, appear to be more stable against variations of $\Lambda$ compared to results of the stochastic rotational method.

The one-dimensional velocity-field profiles across the channel width are shown in Fig. 4 for two different strengths $g$ of the gravitational force. To avoid significant compressibility effects in the system, we restricted ourselves to Poiseuille flows with maximal velocities of about $25 \%$ of the speed of sound $c=\sqrt{2 k_{B} T}$. The flow field appears to be stable along channel. For twice increased external field, $g^{*}$ $=0.005$ versus $g^{*}=0.0025$, the maximal velocity of Poiseuille flow is doubled. Thus, as follows from Eq. (5), the viscosity of solvent for these two runs is the same.

The next two figures reveal the dependence of the velocity profiles on the parameters $\Lambda$ and $\alpha$. Note that when in- 

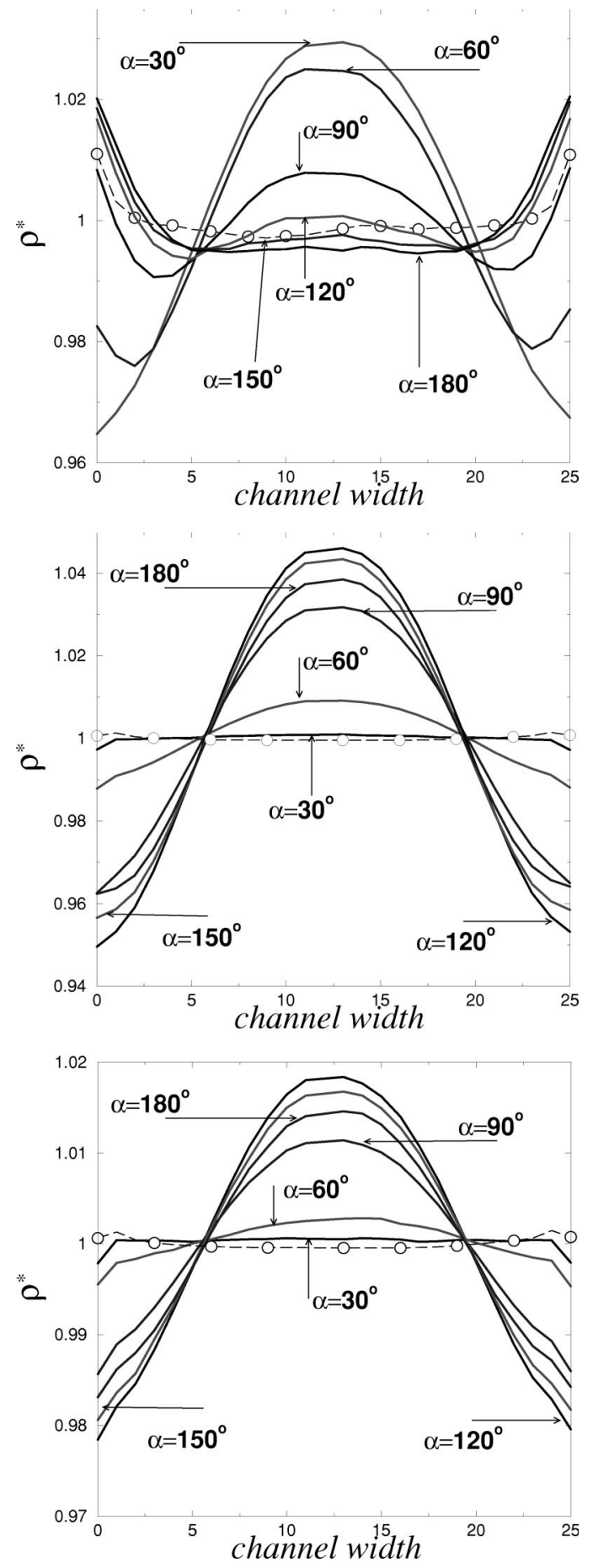

FIG. 3. Normalized density profiles $\rho^{*}=\rho(y) y_{s} / N$ of solvent particles across the channel width for three different values of $\Lambda$. From top to bottom: $\Lambda=0.046,0.58,1.16$. Values of the rotational angles $\alpha$ are given next to the corresponding density curves. Dashed lines with symbols are results of the alternative (random angle and random velocity) collision models; see text for more details.

creasing either $\alpha$ (see Fig. 5) or $\Lambda$ (see Fig. 6), the flow velocity first increases, and then decreases again. Thus, via variation of $\alpha$ and $\Lambda$, the viscosity of solvent can be tuned within a broad range of values. This is explicitly shown in

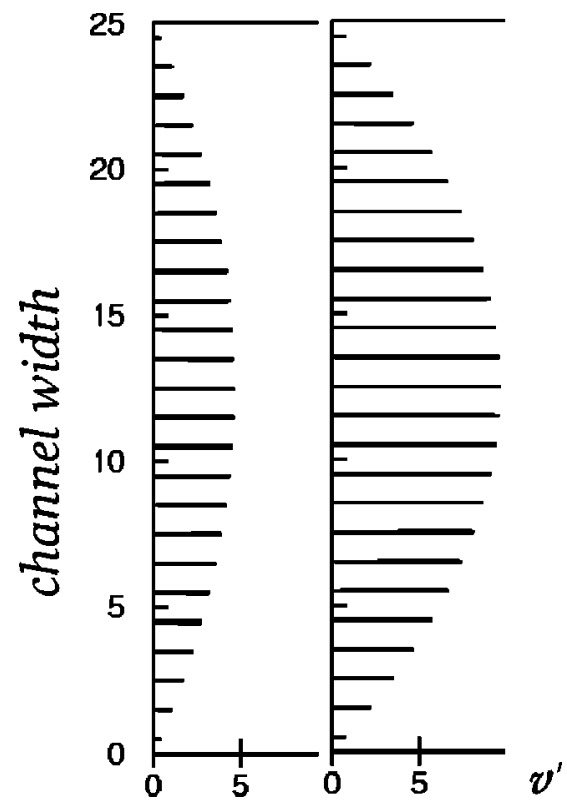

FIG. 4. Rescaled velocity profiles, $v^{\prime}=v / \sqrt{k_{B} T} \times 10^{2}$, of Poiseuille flow for $\alpha=90^{\circ}, \Lambda=0.58$ and two dimensionless gravitational force strengths, $g^{*}=0.0025$ (left plot) and $g^{*}=0.005$ (right plot).

Fig. 7, where the kinematic viscosity $\nu=\eta / \rho$ is plotted as a function of the rotational angle $\alpha$. There is a global minimum of $\nu$ at $\alpha=90^{\circ}$ for intermediate and large values of $\Lambda$. However, for small $\Lambda$ the viscosity $\nu(\alpha)$ is a monotonically increasing function of $\alpha$. Our results are in good qualitative agreement with the results of Ref. [13] obtained for the 2D case. Note that there is no explicit $\alpha$-dependence of the random angle and the random velocity methods. Both of these algorithms result in an identical value for the solvent viscosity, plotted as a horizontal line for different values of $\Lambda$ in Fig. 7.

Figure 8 represents the variation of the Reynolds number Re with parameter $\alpha$. The minima of $\nu$ in Fig. 7 correspond

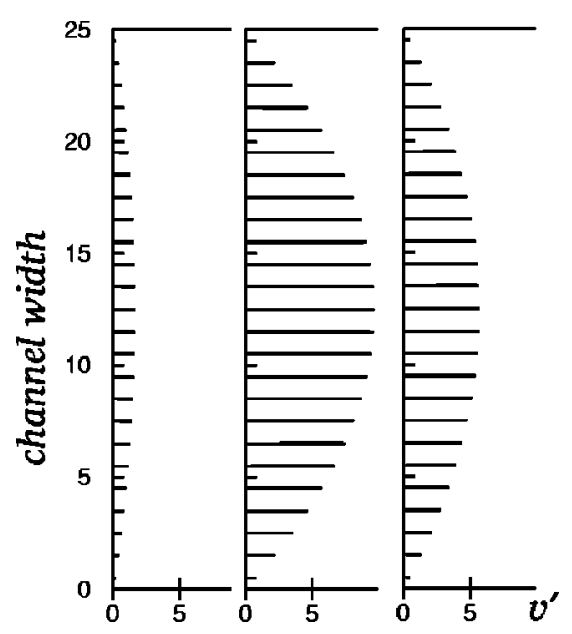

FIG. 5. Rescaled velocity profiles, $v^{\prime}=v / \sqrt{k_{B} T} \times 10^{2}$, of Poiseuille flow for $\Lambda=0.58, g^{*}=0.005$ and three different values of $\alpha$. From left to right: $\alpha=30^{\circ}, 90^{\circ}, 150^{\circ}$. 


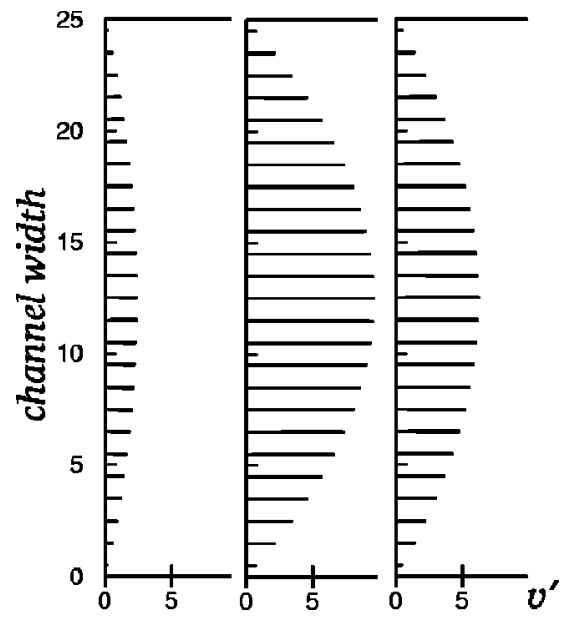

FIG. 6. Rescaled velocity profiles, $v^{\prime}=v / \sqrt{k_{B} T} \times 10^{2}$, of Poiseuille flow for $\alpha=90^{\circ}, g^{*}=0.005$ and three different values of $\Lambda$. From left to right: $\Lambda=0.046,0.58,1.16$.

to maxima of $\operatorname{Re}=\rho v_{\max } L_{y} / \eta$ in Fig. 8. The intriguing nonmonotonic dependence of the Reynolds number Re on the rescaled mean free path of the solvent particles, $\Lambda$, is drawn separately in the inset of Fig. 8. The parameter Re achieves its maximal value $\mathrm{Re} \approx 90$ at $\Lambda \approx 0.35$. the main implication of Fig. 8 is the possibility to achieve sufficiently high Reynolds numbers if appropriate system parameters, rotational angle $\alpha$ and free path length $\Lambda$, are chosen. Our alternative (random angle and random velocity) collision algorithms give Reynolds numbers of the same magnitude as the stochastic rotational method. However, they turn out to be less suitable to produce large-Reynolds-number flows for a given channel geometry, as a function of $\Lambda$, compared to flows produced by stochastic collisional method; see dashed line

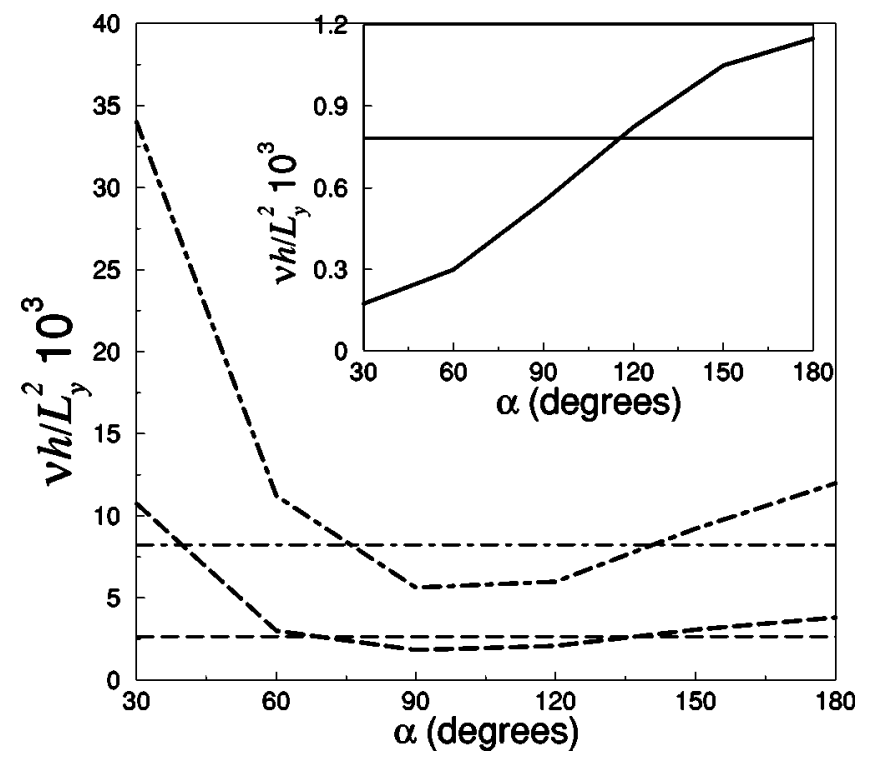

FIG. 7. Rescaled kinematic viscosity $\nu h / L_{y}^{2}$ versus rotational angle $\alpha$ for $g^{*}=0.005$ and three different mean free paths $\Lambda$. Solid curve, $\Lambda=0.046$ (see inset); dashed curve, $\Lambda=0.58$; dashed-dotted curve, $\Lambda=1.16$. Thin horizontal lines correspond to the alternative collision (random angle and random velocity) models.

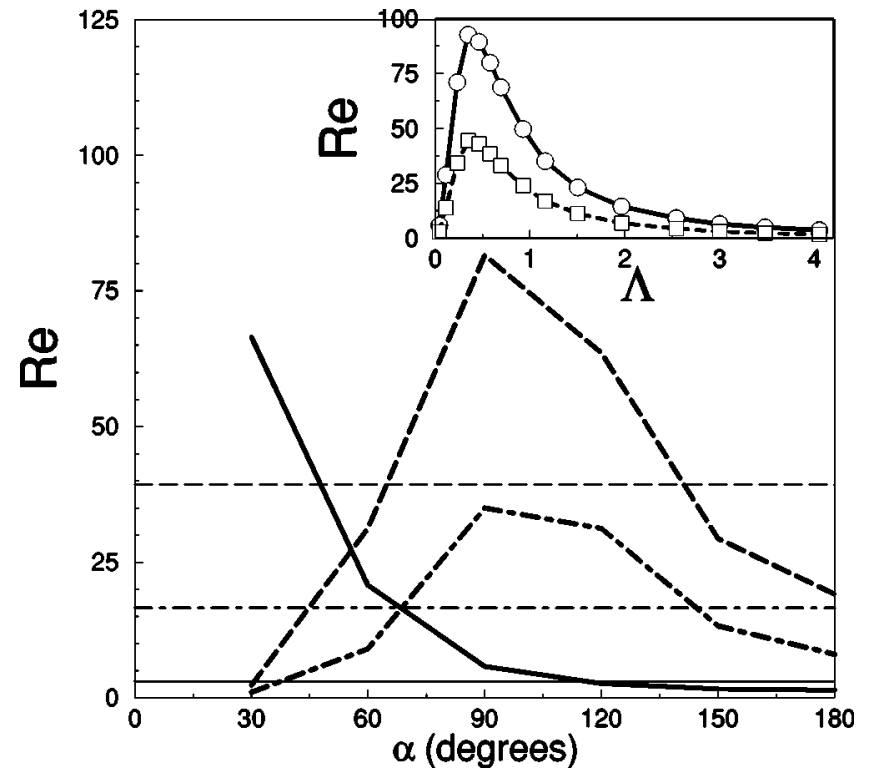

FIG. 8. Reynolds number Re versus rotational angle $\alpha$ for $g *$ $=0.005$ and three different mean free paths $\Lambda$, compare Fig. 7 . Solid curve, $\Lambda=0.046$ (see inset); dashed curve, $\Lambda=0.58$; dashdotted curve, $\Lambda=1.16$. The inset shows $\operatorname{Re}$ as a function of mean free path $\Lambda$ for $\alpha=90^{\circ}$ and $g^{*}=0.005$. Solid line, stochastic rotation method; dashed line, alternative collision methods.

with symbols in the inset of Fig. 8. This has the advantage that large Reynolds-number flows can be studied without having to increase the system size and thereby the number of solvent particles too much.

Next we consider the behavior of the kinematic viscosity $\nu$ with varying mean-free-path length $\Lambda$. It is seen from the inset of Fig. 9 that $\nu$ is nearly constant in the limit of small free path length of solvent particles ( small $\Lambda$ ), approaching the value $\nu^{*}=\nu h /\left(L_{y}^{2}\right)=5 \times 10^{-4}$ for $\Lambda \rightarrow 0$. This finding is in qualitative agreement with the prediction of an analytical theory for two-dimensional stochastic fluid model [13] in the small $\Lambda$ limit,

$$
\nu=\frac{l_{c}^{2}}{12 h}[1-\cos \alpha] .
$$

which yields $\nu^{*}=1.3 \times 10^{-4}$ for $\alpha=90^{\circ}$. The asymptotic behavior of simulated $\nu$ at large $\Lambda$ is phenomenologically well described by the analytical result of Ref. [11],

$$
\nu=\frac{\Lambda^{2}}{18 h} \frac{3\left(1-e^{-M}\right)+2 M}{e^{-M}-1+M}
$$

see dashed line in Fig. 9.

The dependence of the Reynolds number and the kinematic viscosity on the mean particle number $M$ are plotted in Fig. 10. The denser the fluid ( $M$ is also a measure of fluid density), the larger the Reynolds number becomes. 


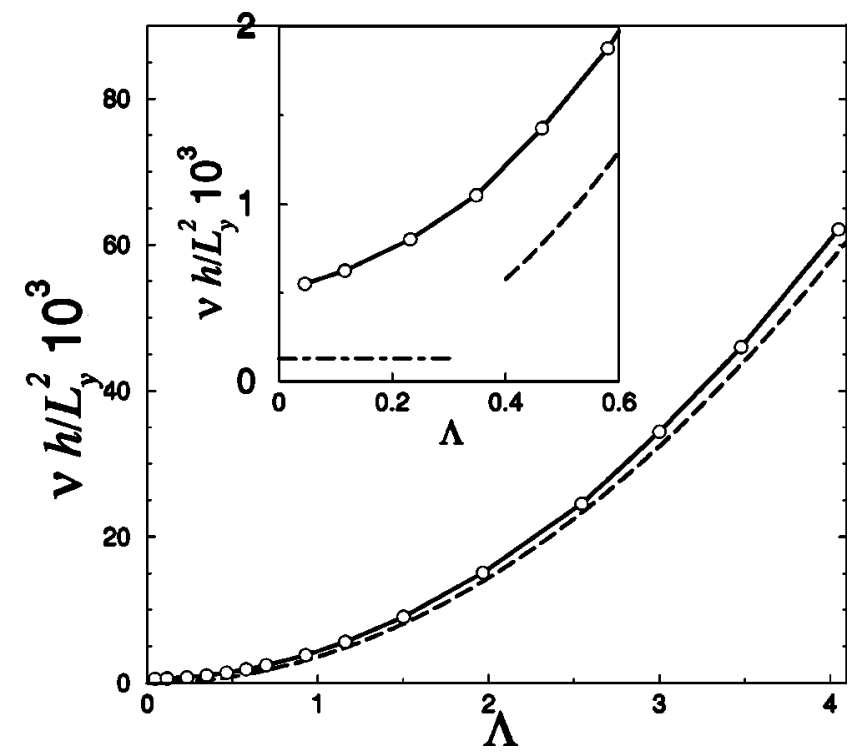

FIG. 9. Rescaled kinematic viscosity $\nu h / L_{y}^{2}$ as a function of dimensionless mean free path $\Lambda$ for $\alpha=90^{\circ}$ and $g^{*}=0.005$. Solid lines with symbols, simulation result; dashed line, analytic result for two-dimensional flow from Ref. [11]. The inset shows a detailed picture for small values of $\Lambda$. The dashed-dotted horizontal line in the inset is the prediction of Eq. (6) for two-dimensional flow and small $\Lambda$.

\section{B. Flow around a spherical obstacle}

We now apply the multiparticle-collision method to the problem of solvent flow past a spherical obstacle. This flow geometry is well documented in many textbooks of fluid mechanics. The applicability of the current mesoscopic solvent model in 2D simulations of flow around obstacles has been demonstrated in Ref. [12] for a wide range of Reynolds

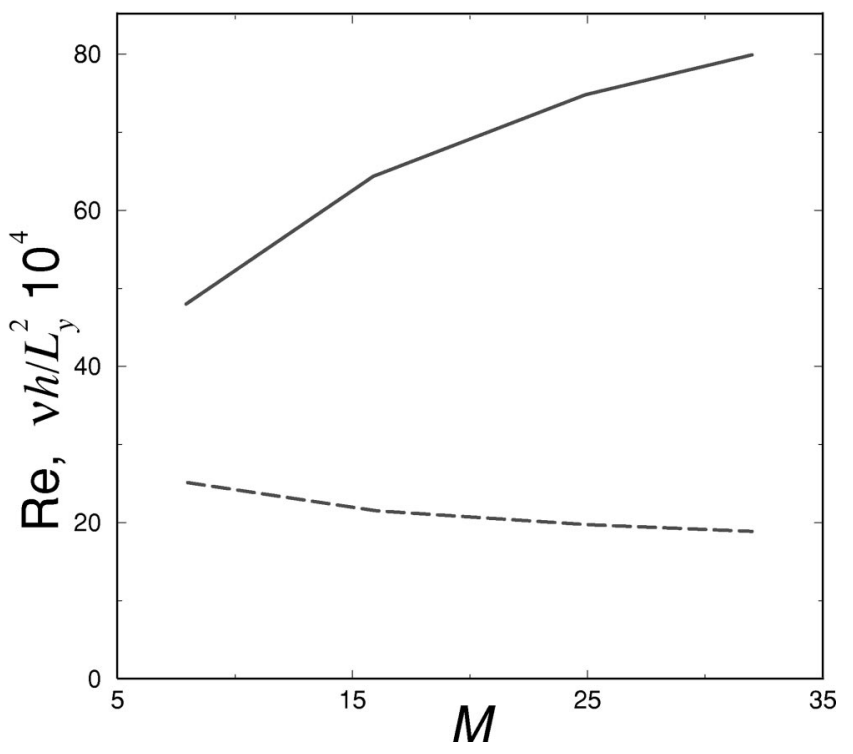

FIG. 10. Reynolds number Re (solid line) and rescaled kinematic viscosity $\nu h / L_{y}^{2}$ (dashed line) as a function of average particle number $M$ in a cell for $g^{*}=0.005$ and $\Lambda=0.58$.

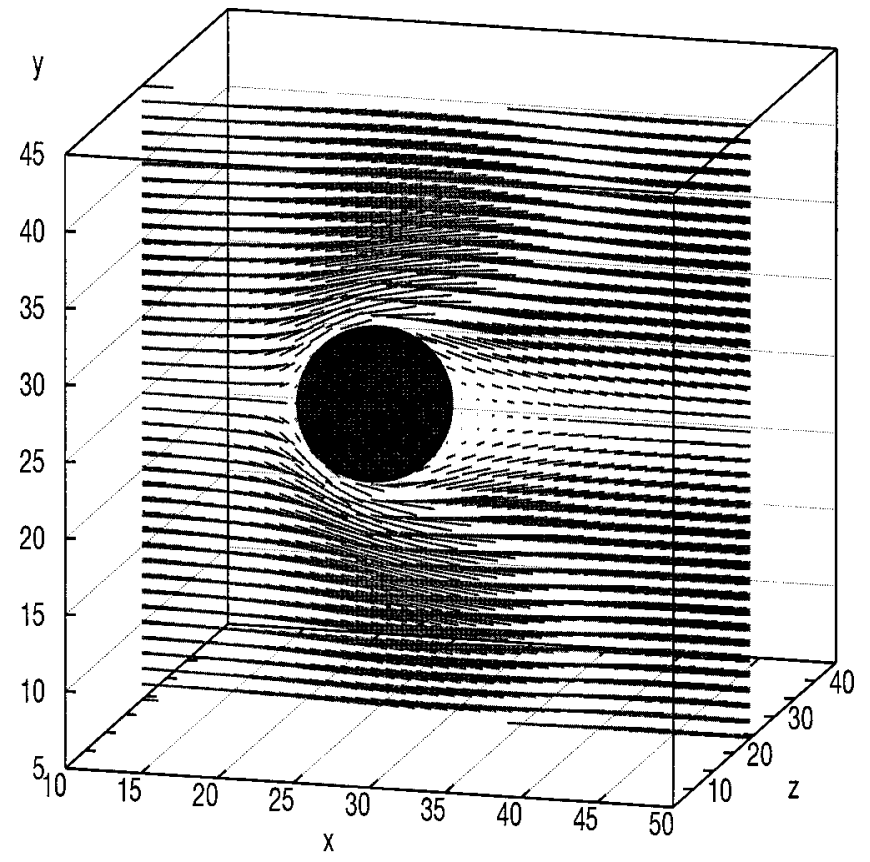

FIG. 11. Typical snapshot for 3D flow over sphere. Only the velocity profile across the middle of $z$ plane of flow channel is shown.

numbers. The high accuracy of this method for flow past spheres in three-dimensional flow is illustrated below.

The simulations are performed in channel of volume $150 \times 100 \times 100$, with $10^{7}$ particles and blockage ratio $D / L_{y}=0.2$. We use a planar (constant) velocity-field profile in the channel, i.e., we replace the bounce-back condition by an ordinary elastic collision of solvent particles with the channel walls.

A typical 3D picture of flow around a sphere is depicted in Fig. 11. The flow is driven from left to right. For the sake of clarity, only the middle z-plane velocity field is shown. Each segment in Fig. 11 represents the orientation of the average velocity of the solvent particles in the cell located at the left end of the segment. The length of segments is proportional to the magnitude of the velocity. More detailed pictures of the flow at the rear part of sphere for different Reynolds numbers $\operatorname{Re}_{D}=\rho v_{\text {max }} D / \eta$, where $D$ is the sphere diameter, are shown in Fig. 12. The increase of $\operatorname{Re}_{D}$ produces a swelling of the wake area behind the sphere, see Fig. 12 from top to bottom. At higher values of $\mathrm{Re}_{D}$ a closed steady recirculation region of vortices occurs behind the obstacle. This is visible as a development of two symmetric vortices in Fig. 12. The length of this steady wake, $l_{w}$, defined as distance from the rear point of the sphere to the point of zero velocity behind the obstacle, is plotted in Fig. 13 versus $\operatorname{Re}_{D}$. The dependence $l_{w}\left(\mathrm{Re}_{D}\right)$ is monotonic, and is in very good quantitative agreement with the experimental results of Ref. [17] and the theoretical prediction of Ref. [18].

As a last application of mesoscopic solvent model, the influence of the embedded sphere on the effective viscosity of the solvent is investigated. For such a flow with an obstacle in channel, the application of Eq. (5) defines an effec- 

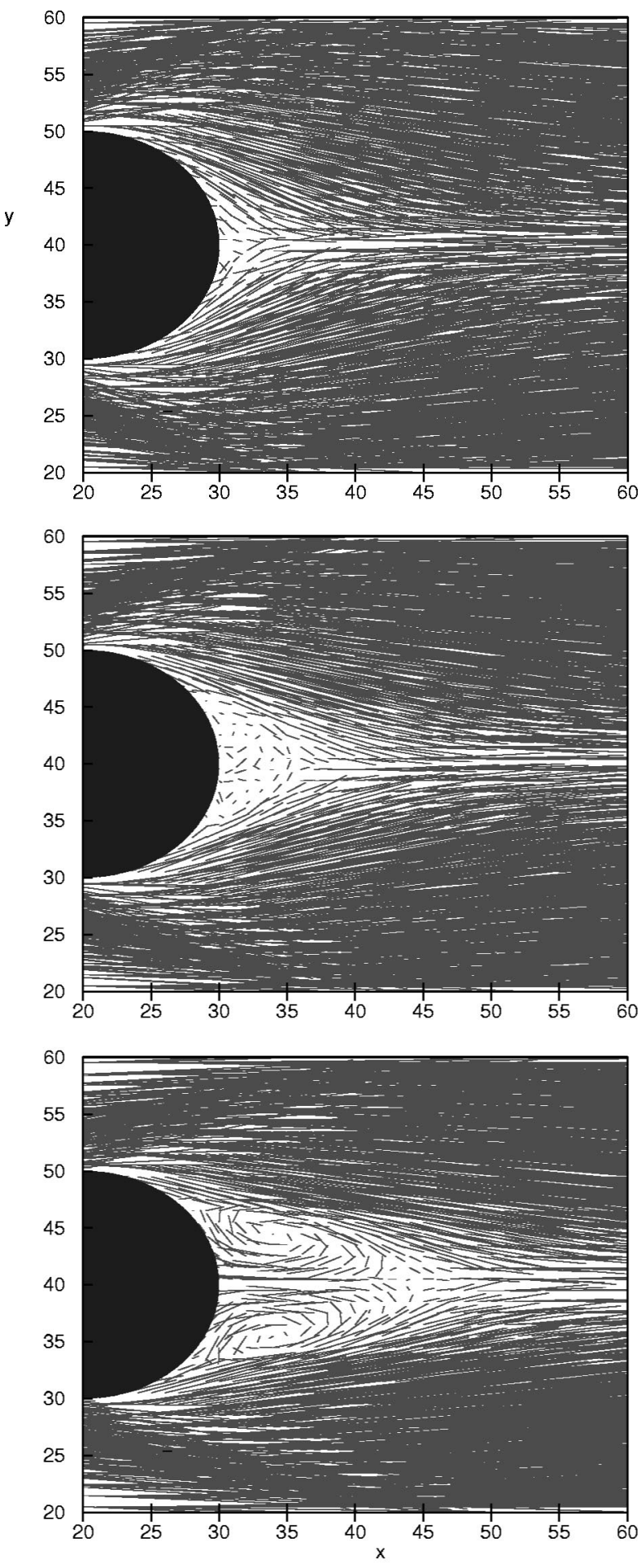

FIG. 12. Velocity field of solvent flow past sphere. The system parameters are: $M=8, g^{*}=0.005, \alpha=90^{\circ}$. From top to bottom: $\operatorname{Re}_{D}=24(\Lambda=1.8), \operatorname{Re}_{D}=40(\Lambda=0.9), \operatorname{Re}_{D}=76(\Lambda=0.35)$.

tive viscosity $\eta_{e f f}$, which is determined not only by $\eta$, but also by the flow geometry. Obviously, without the obstacle, $\eta_{\text {eff }}=\eta$. We have measured the effective solvent viscosity $\eta_{\text {eff }}$ of flows with and without obstacle at fixed other system parameters. The decrease of the flow rate, seen in Fig. 14,

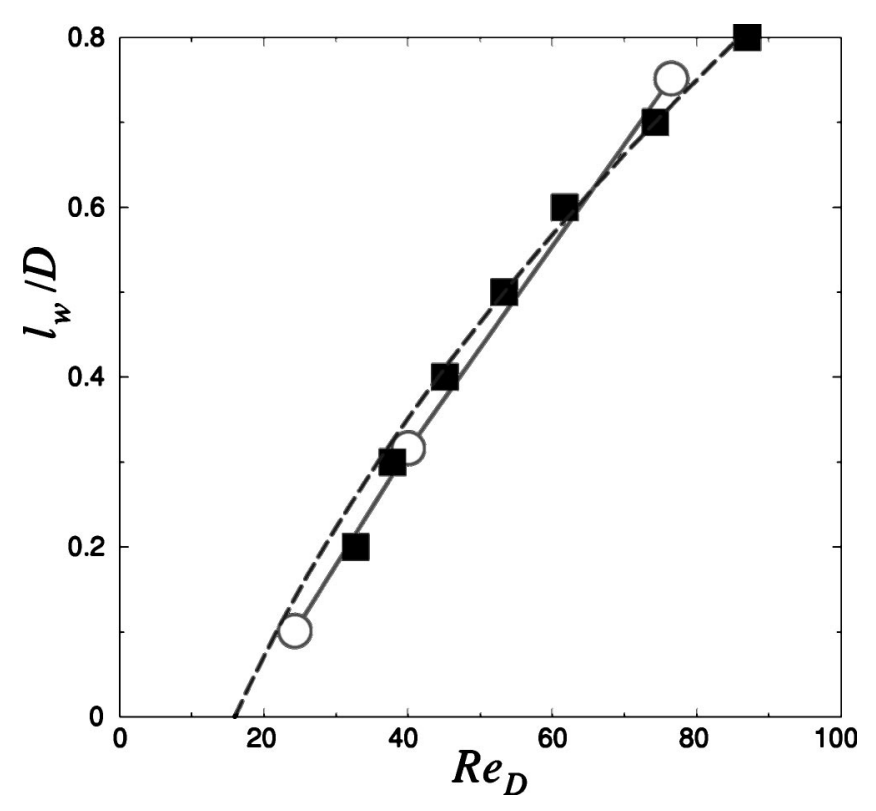

FIG. 13. The length of the stationary wake behind a sphere as a function of the Reynolds number $\mathrm{Re}_{D}$. Filled squaresexperimental data from Ref. [17], theoretical result from Ref. [18] [see theoretical Eq. (16.28) there]; open circles connected with solid line-simulation results of current work.

indicates the increase of the effective solvent viscosity, when a sphere is embedded.

\section{CONCLUSIONS}

We have investigated in this paper a mesoscopic simulation method - multiparticle-collision dynamics — in three dimensions, and its application to flow in a planar channel and to flow past a sphere. The advantage of a gravitationally driven flow over a forced flow in calculating the solvent viscosity is shown. We exploit two alternative algorithms for updating the particle velocities in the collisional step. Both of these algorithms, the random angle and random velocity methods, yield almost identical results for the velocity and density profiles of solvent particles at other fixed system parameters. Compared to the stochastic rotational method of Refs. [10,11], the alternative collision methods yield more stable and homogeneous particle density profiles across the width of the flow channel. When the computational efficiency is concerned, the random velocity method, which is free from the rotations of velocities (see the Appendix), appears to take less simulation time than other algorithms for the stochastic collision procedure. A rough estimate made through simulations of solvent flow between walls shows a gain about $40 \%$ in computational time. On the other hand, our simulations reveal a disadvantage of the random velocity method for larger Reynolds flows. The reason for that is the difficulty to reduce sufficiently the viscosity of the solvent by tuning the only free parameter $\Lambda$ in the random velocity method. For this purpose the second free parameter of the solvent, an explicit rotational angle $\alpha$, turns out to be very convenient. That is why, for solvent flows around sphere, where the most interesting area of investigations is larger 

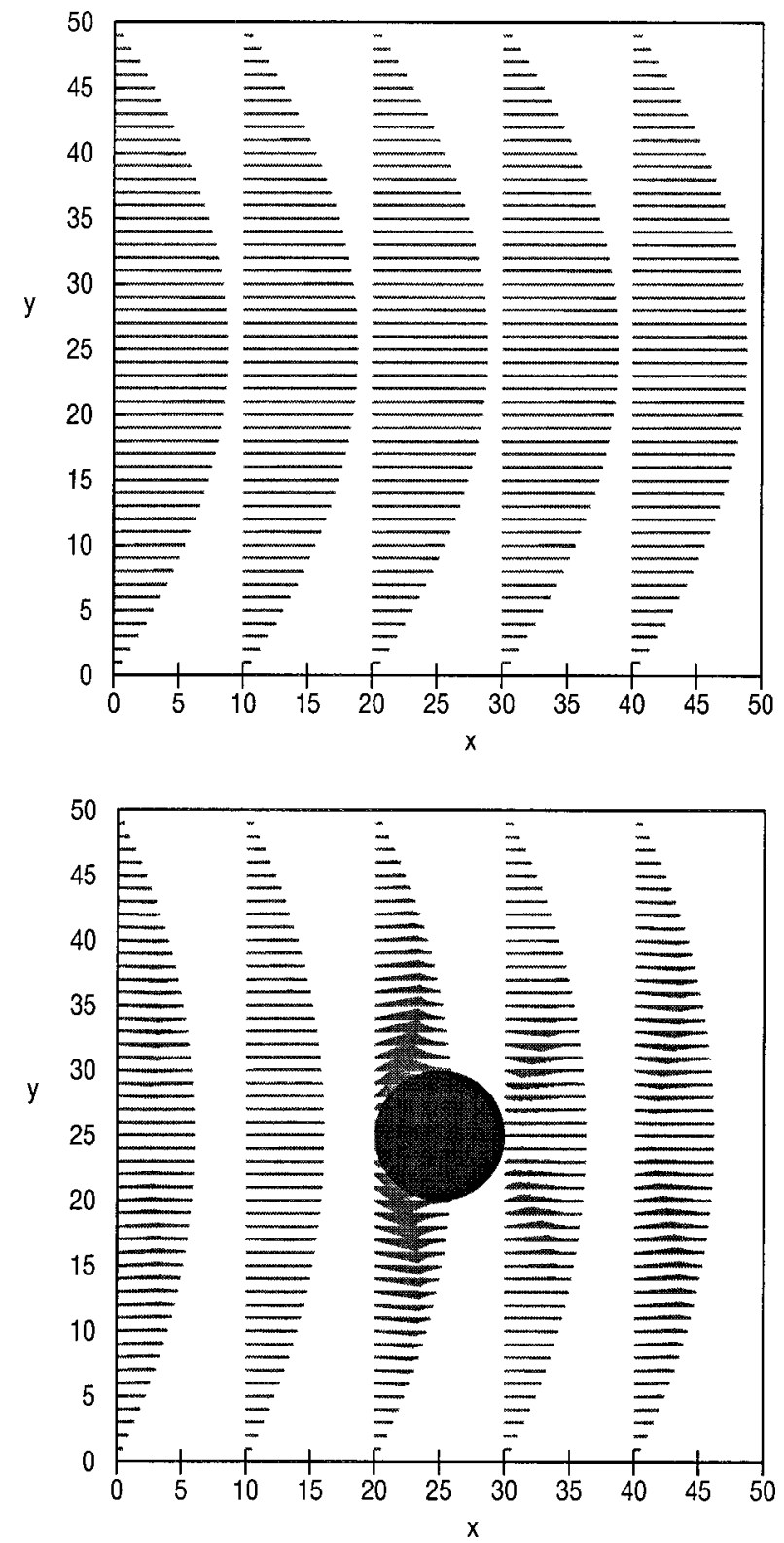

FIG. 14. The increase of the viscosity $\eta$ of system with embedded obstacle as seen from the drop of the flow velocity. Upper figure, $\operatorname{Re}_{D}=50$; bottom figure, $\operatorname{Re}_{D}=25$. The other parameters are $g^{*}=0.005$ and $\Lambda=0.46$.

Reynolds numbers, we employ the stochastic rotational method.

The results obtained show the efficiency of this mesoscopic model in applications to a wide range of physical systems, where solvent dynamics is believed to play an essential role. The main efficiency of the scheme derives from the fact that the solvent-solvent interactions are considered only at discrete time intervals and modeled by a multiparticlecollision rule. The collision model can easily be extended to treat more complex systems, such as charged colloid suspensions. To simulate the hydrodynamic interaction between all charged entities in such systems, motion of small ions have

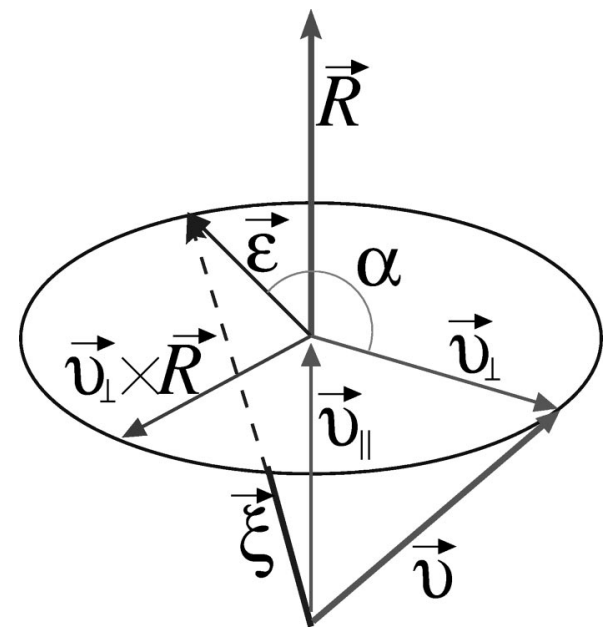

FIG. 15. Rotation of a vector $\vec{v}$ around a direction given by the unit vector $\vec{R}$.

to be coupled to solvent dynamics through stochastic collision.

\section{ACKNOWLEDGMENTS}

We thank D. M. Kroll and A. Lamura for helpful comments.

\section{APPENDIX: ROTATION OF A 3D VECTOR AROUND A RANDOM DIRECTION}

We describe here the rotation procedure of a vector $\vec{v}$ around a random vector $\vec{R}$. The latter can be considered as a vector generated from the uniform distribution on the spherical surface of radius $R=1$. This random vector $\vec{R}$ has the components

$$
R_{x}=\cos \varphi \sqrt{1-\rho^{2}}, \quad R_{y}=\sin \varphi \sqrt{1-\rho^{2}}, \quad R_{z}=\rho,
$$

where $\varphi=2 \pi R_{1}, \rho=2 R_{2}-1, R_{1}$ and $R_{2}$ are uncorrelated random numbers from a uniform distribution in the interval $[0,1]$.

The rotation of $\vec{v}$ around $\vec{R}$ by angle $\alpha$ is sketched in Fig. 15. The illustrated rotation transform the vector $\vec{v}=\vec{v}_{\perp}$ $+\vec{v}_{\|}$onto the vector $\vec{\xi}=\vec{\varepsilon}+\vec{v}_{\|}$, where $\vec{v}_{\perp} \vec{R}=0$ and $\vec{v} \| \vec{R}$ $=|\vec{v} \||$. In order to define the vector $\vec{\xi}$ one has to calculate the three-dimensional components of the vector $\vec{\varepsilon}$. It is straightforward to split the vector $\vec{\varepsilon}$ into two components lying in the plane that is perpendicular to the vector $\vec{R}$,

$$
\vec{\varepsilon}=\vec{v}_{\perp} \cos \alpha+\left(\vec{v}_{\perp} \times \vec{R}\right) \sin \alpha .
$$

Here $\vec{v}_{\perp} \times \vec{R}$ is the vector product of $\vec{v}_{\perp}$ and $\vec{R}$. 
[1] P. Pincus, in Structure and Dynamics of Strongly Interacting Colloids and Supramolecular Aggregates in Solution, Vol. 369 of NATO Advanced Study Institute, Series C: Mathematical and Physical Sciences, edited by S. -H. Chen, J. S. Huang, and P. Tartaglia, (Kluwer Academic, Dordrecht, 1992).

[2] E. Allahyarov and H. Löwen, Phys. Rev. E 63, 041403 (2001).

[3] F. Otto and G.N. Patey, Phys. Rev. E 60, 4416 (1999); J. Chem. Phys. 112, 8939 (2000).

[4] M. Kinoshita, S. Iba, and M. Harada, J. Chem. Phys. 105, 2487 (1996).

[5] S. Marcelja, Colloids Surf., A 129-130, 321 (1997).

[6] S. Marcelja, Langmuir 16, 6081 (2000).

[7] V. Kralj-Iglic and A. Iglic, J. Phys. II 6, 477 (1996); I. Borukhov, D. Andelman, and H. Orland, Phys. Rev. Lett. 79, 435 (1997); E. Trizac and J.-L. Raimbault, Phys. Rev. E 60, 6530 (1999).

[8] G.R. McNamara and G. Zanetti, Phys. Rev. Lett. 61, 2332 (1988); F.J. Higuera and J. Jimenez, Europhys. Lett. 9, 663 (1989).

[9] E.S. Boek, P.V. Conveney, H.N.W. Lekkerkerker, and P. van der Schoot, Phys. Rev. E 55, 3124 (1997); I. Pagonabarraga,
M.H.J. Hagen, and D. Frenkel, Europhys. Lett. 42, 377 (1998). [10] A. Malevanets and R. Kapral, J. Chem. Phys. 110, 8605 (1999).

[11] A. Malevanets and R. Kapral, J. Chem. Phys. 112, 7260 (2000).

[12] A. Lamura, G. Gompper, T. Ihle, and D.M. Kroll, Europhys. Lett. 56, 319 (2001).

[13] T. Ihle and D.M. Kroll, Phys. Rev. E 63, 020201(R) (2001).

[14] It is also used to be called "no-slip" or "stick" boundary conditions. Due to this condition, the velocity of a particle which hits the wall is totally reversed.

[15] Statistical Mechanics of Nonequilibrium Liquids, edited by D. Jeans and G. P. Morriss (Academic Press, London, 1990).

[16] In order to keep the system temperature constant during the simulation time, a velocity scaling procedure is applied to the relative particle velocities $\vec{\xi}_{i}(t), i=1,2, \ldots, N$.

[17] S. Taneda, Rep. Res. Inst. Appl. Mech. (Kyushu Univ.) 4, 99 (1956).

[18] Viscous Flows, edited by S. W. Churchill, Butterworths series in chemical engineering Vol. 1 (Butterworth, Stoneham, 1988). 